\title{
CELL REACTIONS WITH BIOMATERIALS: THE MICROSCOPIES
}

\author{
A.S.G. Curtis* \\ Centre for Cell Engineering, University of Glasgow, Glasgow G12 8QQ, Scotland, U.K.
}

\begin{abstract}
The methods and results of optical microscopy that can be used to observe cell reactions to biomaterials are Interference Reflection Microscopy (IRM), Total Internal Reflection Fluorescence Microscopy (TIRFM), Surface Plasmon Resonance Microscopy (SPRM) and Förster Resonance Energy Transfer Microscopy (FRETM) and Standing Wave Fluorescence Microscopy. The last three are new developments, which have not yet been fully perfected. TIRFM and SPRM are evanescent wave methods. The physics of these methods depend upon optical phenomena at interfaces. All these methods give information on the dimensions of the gap between cell and the substratum to which it is adhering and thus are especially suited to work with biomaterials. IRM and FRETM can be used on opaque surfaces though image interpretation is especially difficult for IRM on a reflecting opaque surface. These methods are compared with several electron microscopical methods for studying cell adhesion to substrata. These methods all yield fairly consistent results and show that the cell to substratum distance on many materials is in the range 5 to $30 \mathrm{~nm}$. The area of contact relative to the total projected area of the cell may vary from a few per cent to close to $100 \%$ depending on the cell type and substratum. These methods show that those discrete contact areas well known as focal contacts are frequently present. The results of FRETM suggest that the separation from the substratum even in a focal contact is about $5 \mathrm{~nm}$.
\end{abstract}

Key Words: Interference reflection, total internal reflection fluorescence, resonance energy transfer, surface plasmon resonance, cell contacts.

"Address for correspondence:

A.S.G. Curtis

Centre for Cell Engineering, University of Glasgow, Glasgow G12 8QQ, Scotland, U.K.

Telephone Number: +44 1413305147

FAX Number: +44 1413303730

E-mail: a.curtis@bio.gla.ac.uk

\section{Introduction}

Cells often live, both in the body and in the cell culture laboratory, in transient or long term contact and adhesion with surfaces. These surfaces may be those of biomaterials, implanted or natural. These surfaces are often patterned with chemical or topographic features, which may increase adhesion. Control of adhesion is an important aim for those using biomaterials. For these reasons we need to understand how cells adhere to such materials especially when they have received surface treatments. One type of knowledge is derived from images of the cell's contact with the surface in life. This knowledge may tell us whether the cell confines its adhesive contacts to small parts of the surface or whether they are general, whether they are very close contacts and thus probably very strong or whether they are ones where the cell does not approach very close to the surface and thus perhaps weak. In the second case we can speak of a gap existing in the contact. There are two possible reasons for the presence of a gap. The first is that the cell and substratum surfaces are really separated by an intervening medium gap. Obviously this might be so in those regions of the cell where no adhesive contact has been formed but they might also be found where a physically driven adhesion of the secondary minimum type is present (Hunter, 1987).

There is much evidence that many types of cells react to these features in a variety of ways including gene activation and signal transduction processes (Curtis and Wilkinson, 1998). One controversial possibility is that the cells react to topography rather than to chemical differences. While this is not yet proven or disproven the possibility has been discussed with evidence being adduced in favour of the topographic hypothesis.

Appropriate optical methods for examining cell adhesion

In this paper I shall concentrate on discussion of the methods for visualising the contact side of the cell and on the information on contact reactions these methods supply. In a sense the relevant microscopies are ones directed at the underworld. Most of these techniques can only be applied in situations in which the cell is attaching to a surface with a well-defined interface which provides a clear reference surface for image formation and often for calibration as well. Thus most of the methods cannot be applied to cell to cell contacts but are ideal for many aspects of biomaterial research.

In practice these requirements confine the surfaces we can use experimentally to transparent ones with a flat sur- 


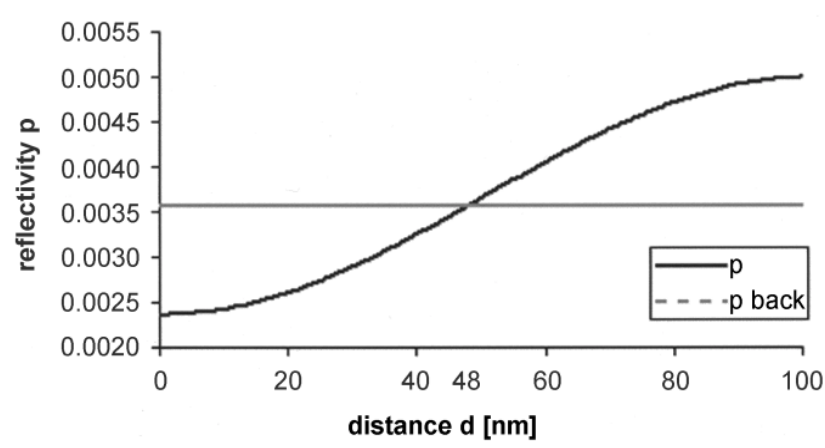

Figure 1. The relationship between cell-glass separation distance in nanometres and the intensity of the IRM image. For comparison the light reflected at a glass:culture medium interface (flat line) is shown and for the set of conditions chosen the image changes from darker than background to brighter than background at a separation of $48 \mathrm{~nm}$.

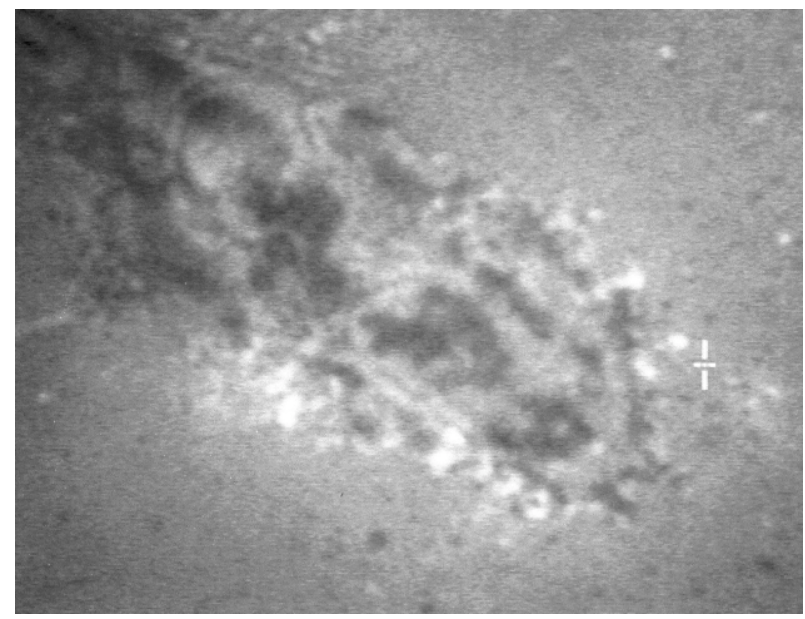

Figure 2. An IRM image of an epitenon cell ten minutes after plating out. Note the faintly seen parallel interference bands at the top-left hand part of the image. These probably arise from a sloping "overhang" of the cell. Note also that the cell does not show the "classical" focal contacts.. Scale $1 \mathrm{~cm}=0.8 \mu \mathrm{m}$.

face, e.g. glasses, silica, many polymers, and materials with large transparent crystals and liquids immiscible with culture media. Fortunately many standard materials for cell culture, though not so many for use as biomaterials, have these properties. Surface plasmon resonance microscopy can only be used on thin metallic films though they may be coated with monolayers of protein etc. Interference reflection microscopy depends on reflections at a surface and is suited to use on very flat materials of reasonable reflectivity whether opaque or transparent and is perhaps the microscopy most suited to biomaterials research.

One important piece of information that has been sought is the distance between the adhering side of the cell and the substratum. This information gives information on the contact area of the cells with the substratum and the closeness of approach of cell to substrate. Can we for example correlate the closeness of that approach with the strength of the adhesion? This measurement depends upon the assumption that the plasmalemma is a boundary at which there is a steep change in refractive index over a very short distance, approximately $7 \mathrm{~nm}$. The reasons for this conclusion are discussed below. It also should be noted as Curtis (1964) remarked that the range of refractive indices that can assigned to the plasmalemma and to the intervening medium cannot be varied appreciably without the nature of the calculated image changing from that actually observed.

\section{Optical Methods for Examining the Underside of the Cell in Life \\ Interference Reflection Microscopy (IRM) sometimes known as Reflection Interference Contrast Microscopy}

This method depends upon the interference between light reflected at the surface of the substratum and that reflected at the undersurface of the cell. If there is no gap separating cell and substratum interference does occur and the reflectivity becomes that of light at the biomaterial surface. A curve showing the intensity of reflected light in terms of the distance between cell and substrate is shown in Figure 1 . This presumes that there are no other surfaces of appreciable reflectivity close to either of these surfaces. The observation is carried out with vertically incident or nearly vertically incident light (preferably monochromatic) falling on the contact zone through either an inverted or a conventional microscope. A good Ploem-type fluorescence microscope can be converted to an IRM microscope by replacing one of the dichroic beam splitters with a neutral beam splitter. Addition of an extra field iris in the optical train close to the lamp reduces diffuse light and helps in setting up. High intensity tungsten-halogen, mercury or xenon sources may all be used. It is useful to use a good video camera to observe the image because this allows the light intensity to be reduced to a low level that does not damage the cell. Ultraviolet (UV) and heat filters as well as a set of Neutral Density filters are desirable. As far as I know this type of equipment is not available commercially at present. The Leitz system produced at one time led to false images because it contained a central field stop that removed most of the nearly vertically incident light rays and introduced a type of out-of-focus phase contrast. On the other hand a confocal laser scanning microscope operating in the reflection mode can produce IRM images. It is important to note the remark made earlier about the need for absence of other nearby surfaces that may reflect light and lead to image complexity. So cells being examined by this system should be placed with a fairly deep layer of medium above (or below) them so that in focus reflections are not obtained from the other side of the system. It is highly probable that some users of the technique have not taken this precaution.

The disadvantages of this type of microscopy are, that it has poor lateral resolution and that because of the nearly flat part of the reflectivity curve (Fig. 1) between 0 and 10 
$\mathrm{nm}$ separation distance contacts in this close and interesting range cannot be measured accurately. A further problem, that can be guarded against, is that reflections from deeper parts of the cell or the other side of the cell are obtained. Such reflections will normally be higher order reflections and thus coloured if white light is used. Such contributions are often seen on the thinner parts of the cell (Fig. 2). The detailed theory of IRM microscopy is covered by Gingell and Todd (1979) and practical advice on the technique is given by Curtis (1989) and by Verschueren (1985).

On the other hand a further advantage for the materials scientist is that very thin deposits on the surface can be detected. DePasquale and Izzard (1987) for example were able to detect trails of material left by cells as they move. These include deposits from the cell where the cell has been but has withdrawn leaving surface contamination behind. Another advantage of the technique for research in cell biology and biomaterials is that surface dirt and contamination can be easily detected.

A potential disadvantage of the method is that if it is slightly incorrectly set-up some internal cytoplasmic components may become imaged. A rough and ready test of correctness of set-up is that the depth of focus of the image is very small indeed being less than $100 \mathrm{~nm}$.

\section{Methods in which an IRM image forms part of the im-} age

When opaque surfaces of reasonable flatness are being studied it is difficult to obtain images of cells growing on the surface. Incident light microscopy using high light intensity or better a sensitive video camera to image the system will often give an image of the cell. This may vary between a rather fuzzy image with low contrast to a very complex image with many fringes on surface of high reflectivity containing both IRM images and reflections from the other side of the cell (Fig. 3). Such images may be useful because they map the other side of the cell as a series of contour fringes.

\section{Four other microscopies}

The next four techniques are evanescent wave methods, though in the case of Förster Resonance Energy Transfer (FRET) microscopy it does not have to use evanescent wave set-ups.

The evanescent wave is generated at an interface when total internal reflection takes place at that interface. The

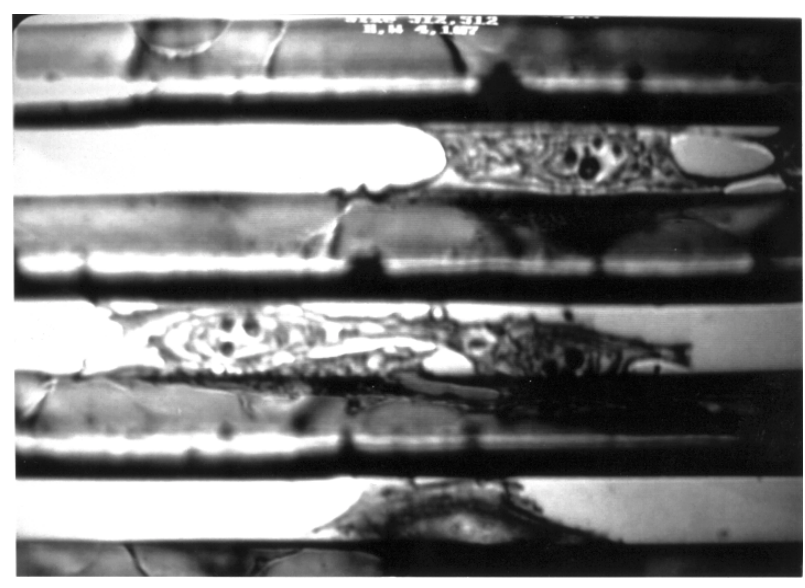

Figure 3. Reflection image of cells growing on silicon groove/ridge topography. The scale is given by the fact that the ridges (in focus) and bright are $10 \mu \mathrm{m}$ wide. The image is complex in nature being composed of interference of the IRM type combined with further interference arising from reflections at the top of the cell.

evanescent wave is in the medium on the opposite side to the surface at which the total internal reflection takes place. The evanescent wave penetrates into the second medium to a depth determined by the angle of incidence, the wavelength and the refractive indices of the media. The energy of the wave declines exponentially with distance from the surface. Evanescent waves can be generated at the surface of a medium such as glass when light is injected at or beyond the total internal reflection angle. Prisms or blocks of glass or silica may be used for this (Fig. 4). An alternative method is to fabricate a waveguide in the substratum, or to use an optic fibre light source. The evanescent wave is not detectable if it does not interact with an object that is capable of absorbing (including fluorescence) or diffracting that wave. But if fluorescing objects or diffracting objects are within about $100 \mathrm{~nm}$ of the interface they may give rise to effects that can be detected from either side of the surface.

Total Internal Reflection Fluorescence Microscopy (TIRFM). Thus if fluorescence is excited in this very thin layer there will be an energy loss in the reflected ray and fluorescence on and very near the surface of the glass detectable as $4 \mathrm{p}$ radiation. The closer to the interface the
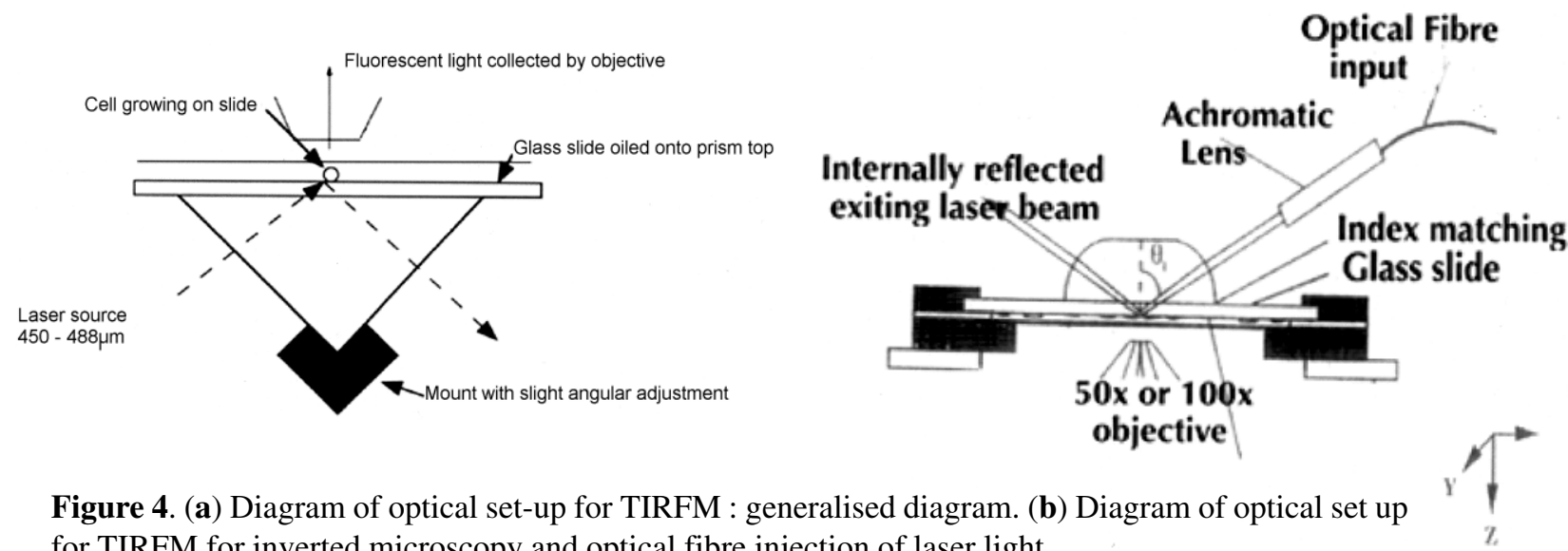

Figure 4. (a) Diagram of optical set-up for TIRFM : generalised diagram. (b)
for TIRFM for inverted microscopy and optical fibre injection of laser light 
location of the fluorescent molecules the more intense the fluorescence because the energy of the evanescent wave falls off exponentially with distance from the surface. Thus, if there is a space $100 \mathrm{~nm}$ or so deep between parts of the cell and the substratum and fluorescent molecules can enter this space fluorescence will be seen. Fluorescent molecules on the top side of the cell or in the space "above" the cell will not be excited being too far from the evanescent wave. The optical set-up is diagrammed in Figure 4a,b. Two early and important papers are those of Axelrod (1981) and Gingell et al. (1985).

The consequence of this system of illumination is that locations where the cell contacts the substratum with a separation of less than $1 \mathrm{~nm}$ will be unilluminated because so few or no fluorescent molecules will have penetrated into that very thin space. If the cell is allowed to settle onto the surface on a fluorescent medium it might be presumed that all regions that are black are ones of very close approach of the cell to the substratum. This presumption is not invariably correct because if the cell has secreted water under its surface this could lift the cell off the substratum but still without fluorescence. In practice TIRFM gives images very similar to those obtained by IRM where the fluorescent areas in TIRFM correspond to the brighter regions in IRM images. In theory it should be capable of detecting very thin layers of medium between the cell and substratum in the 0 to $10 \mathrm{~nm}$ range and while not having good lateral resolution should at least reveal that fluorescent molecules have penetrated into a gap region. Burmeister et al. (1998) carried out an investigation of adhesion to various biomaterials using this method of microscopy.

Surface Plasmon Resonance Microscopy. If a surface is coated with a thin layer of a metal such as a gold, platinum or silver surface plasmons will be present as oscillations of free electrons that propagate along the surface. These oscillations may be excited by coupling to an evanescent wave. The amount of coupling is directly affected by the refractive indices of the materials close on either side of the metal film. In turn the presence of a cell surface modifies the refractive index close to the film. The coupling of the evanescent wave is measured by the drop in totally internally reflected light emerging to a detector. In principle the distance between the metallic surface and the cell is given by the decay length of the plasmon in the direction normal to the surface (the z-direction):

$$
\delta_{e}=[\lambda /(4 \pi)]\left(\left|\varepsilon_{s}{ }^{\prime}+\varepsilon_{a}\right| / \varepsilon_{a}\right)^{1 / 2}
$$

where $\lambda$ is the wavelength of the light, the complex dielectric constant of the metal is given by: $\varepsilon_{s}=\varepsilon_{s}{ }^{\prime}+\mathrm{i} \varepsilon_{s}$ "' and $\varepsilon_{a}$ is the dielectric constant of the medium bounding the metal. By varying the metal and/or wavelength of light the penetration depth of the evanescent field can be changed. Preliminary results show that the system can be used in a microscopical mode. Images appear to resemble those obtained by IRM but should be capable of resolving smaller separations of cell and substratum than $10 \mathrm{~nm}$, though Giebel et al. (1999) only claimed an accuracy of $\pm 10 \mathrm{~nm}$ in a recent paper. Figure 5 shows such an image obtained by SPR.

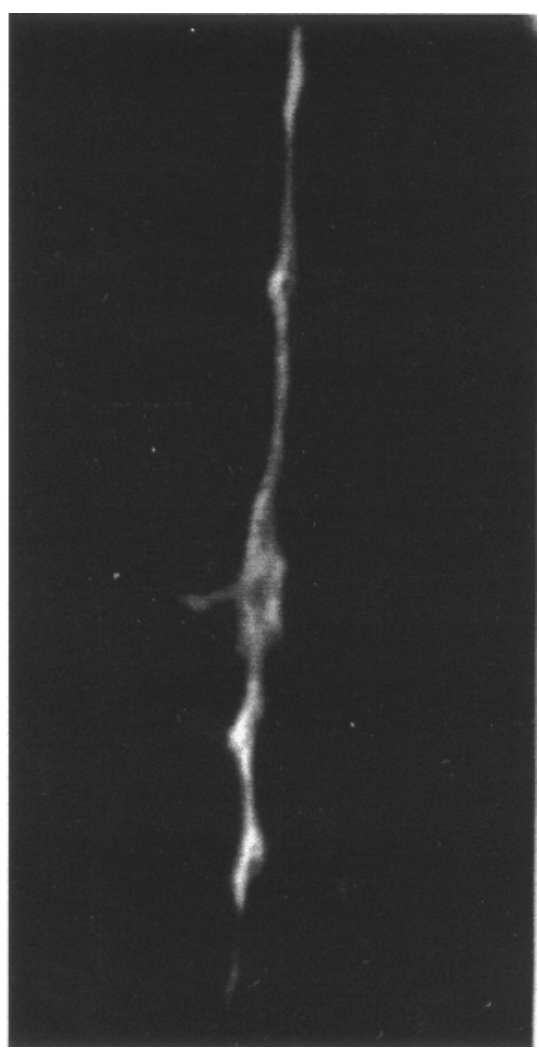

Figure 5. An SPR image of the growth cone region of a neuron. Courtesy of Dr H. Morgan. Scale $1 \mathrm{~cm}=$ $19 \mu \mathrm{m}$.

Förster Resonance Energy Transfer Microscopy (FRET). If two fluorescent centres are located close to each other and the first radiates at the wavelength at which the second centre is excited they may become coupled such that incident light energy absorbed by the first is radiated by the second (Förster, 1949). The energy transfer declines with the distance between the centres to a distance to a six power law. Thus only very close centres will interact detectably. This method has been used fairly widely to examine proximity of two centres or even two molecules in a cell (e.g., Vickery and Dunn, 1999). In principle it should be possible to use it to detect the proximity of a cell with labelled surface molecules to a substratum containing the other type of fluorescent molecule. Usually the donor will be in the substratum. The method has the attraction that it allows measurements of the distance between donor and acceptor in the $0-7 \mathrm{~nm}$ range which is the range not covered by IRM and only at the outer limits by TIRFM.

Though there are practical advantages in exciting the first fluorochrome by use of an evanescent wave in that the need for a very high grade excitation filter is removed it is possible to use direct incident light falling normally on the specimen. The method can be operated in three different modes. First, the secondary fluorescence may be sought. Second, the loss of energy in the primary fluorescence may be sought. Third, a non-fluorescent acceptor may be used in which case the system is run in the same way as in the second case. In all cases there are needs to bind the donor 


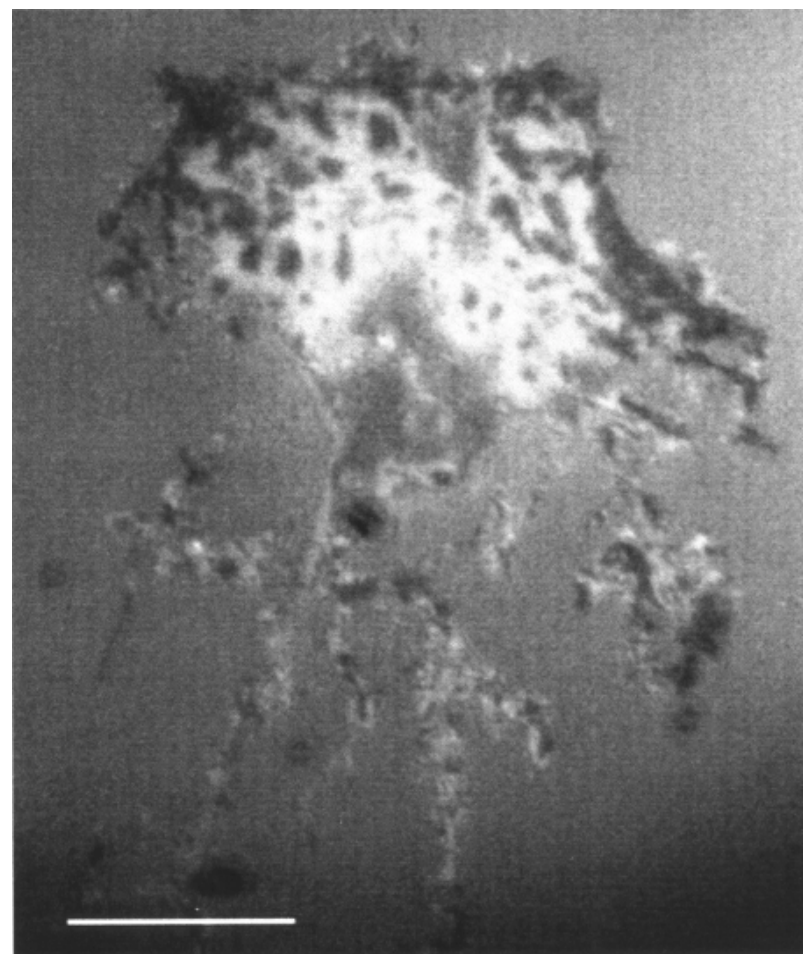

Figure 6. IRM image of an epitenon cell 4 minutes after plating out. Note the complex shape of the developing contact even though the cell was approximately spherical in shape as it settled onto the glass coverslip. Scale bar $=2 \mu \mathrm{m}$.

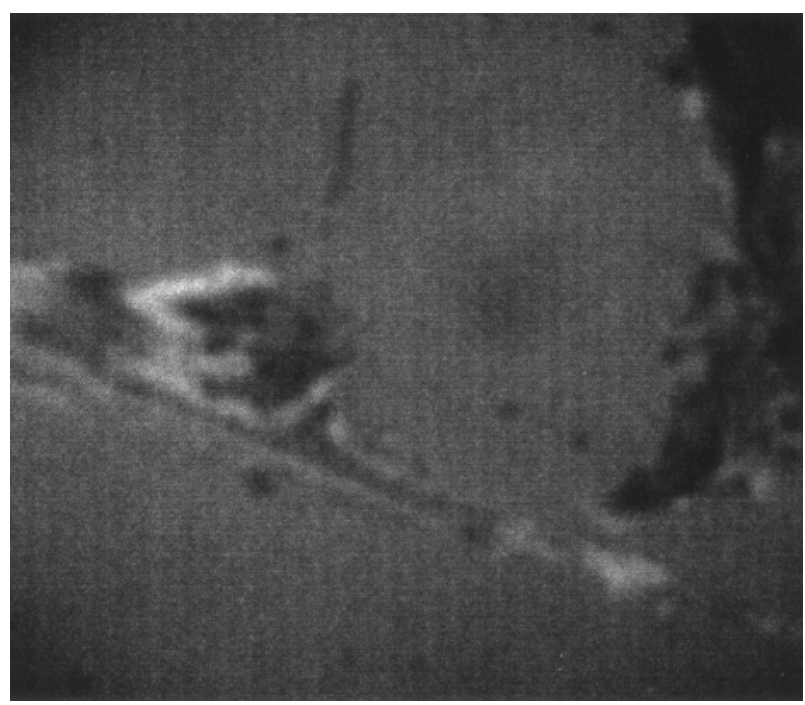

Figure 7. IRM of a rat nodose ganglion growth cone with a microspike (black) running vertically. Note that the microspike does not appear to be connected to the growth cone because it is too far from the surface to be imaged close to the growth cone. Real-time video recordings of this preparation showed rapid changes in the image over the course of one to two seconds. There was also a faster oscillation of the separation distance of the cell under the growth cone. Glial cell on the right. $1 \mathrm{~cm}=1 \mu \mathrm{m}$. and acceptor molecules to the cell and to the substratum.

Using this method Mr P. Clemens working in my laboratory and I have managed to obtain FRET signals from macrophages adhering to a protein surface labeled with fluorescein. The second fluorochrome tetramethylrhodamine was located in the cell surface. A measurement of $5.4 \mathrm{~nm}$ separation was obtained. In principle this method could detect a single molecule binding a cell to a substratum molecule.

Vickery and Dunn (1999) combined FRETM with scanning near-field optical microscopy (SNOM). Though this paper only reports on a model system of multi-layered Langmuir-Blodgett layers it is clear that the system yields good measurements of the distance between the two fluorochromes.

Standing wave fluorescence microscopy. Braun and Fromherz $(1997,1998)$ introduced standing wave fluorescence microscopy. for the examination of cell contacts with a reflecting substratum, such as a silicon layer (beneath its natural oxide covering). In this method a fluorochrome solution is allowed to permeate into any gap under the cell. Optical conditions are set up such that standing waves of light intensity exist in any gap under the cell with maximal excitation of fluorescence at the intensity maxima. Thus if the gap thickness corresponds to the first interference peak, fluorescence is maximal, but if the gap is less in thickness the fluorescence intensity is reduced. In principle this method should work but the originators used a wide cone angle of illumination and a pixel averaging system that probably reduced the accuracy of the system. The reduction in accuracy may explain the rather aberrant results they obtained when cells were grown on laminin.

\section{Electron Microscopical Methods}

Though it might be felt that transmission electron microscopy (TEM) sections of cell contacts would tell us much about cell adhesion to surfaces this has not been especially rewarding because there is little change in the subsequent TEM appearance of the contacts as environmental conditions change adhesion, until the cells fall off. However, two other techniques are more informative. The first is the use of back-scatter electron imaging at various different energy levels see Richards and ap Gwynn (1995), and Richards et al. (1995). It seems to yield images close to IRM images in appearance.

The other technique was introduced by Brightman et al. (1970) but has been little used. This is to permeate tracer molecules that are sufficiently electron-dense to be detected by electron microscopical methods. Particles such as ferritin and micro-peroxidase have been used. The concept is that if these markers penetrate cell contacts rapidly in life these must be an appreciable extracellular space present. This type of experiment reveals that many cell to cell contacts, though not zonulae occludentes, are easily permeated by ferritin. This implies that the separation distance of cell and cell is often greater than $9 \mathrm{~nm}$ over large areas. This result does not preclude the existence of molecules 
bridging between cells but does suggest that the number of such molecules per unit area must be low. As far as I know this type of measurement has not been applied to cell-biomaterial interfaces.

\section{The Contribution from These Methods to Understanding Cell-Biomaterial Interaction}

This section describes the results of experiments and observations that are based on the use of IRM and TIRFM. SRPRM and FRETM are still unperfected and very few results have been reported from their use.

Izzard and Lochner (1976) reported that in many cells the closest approaches of the surface to the substratum were small punctate areas termed by them "focal contacts". Though this concept has led to very many papers using other techniques such as the immunochemical detection of intracytoplasmic proteins such as vinculin and talin there are still some areas of doubt about the accuracy of equating IRM with the immunochemical techniques. First, it is clear that some optical systems have been run such that they penetrate into the cytoplasm. Second, particulate dirt under a cell may become imaged. Third, many images such as those shown here do not demonstrate the type of punctate contact termed a focal contact. It should also be remarked that because of the extreme ability of IRM to detect very thin layers of material it is absolutely essential to grow the cells on ultra-clean surfaces. Usson et al. (1997) used IRM to quantitate cell-biomaterial adhesions. Examination of the forming adhesions shows that the contact area, pattern and closeness of approach may change rapidly with visible events taking place on a scale of one second or less (Figs. 6 and 7).

The first and perhaps the most important contribution to biomaterials studies is that the results are consistent with adhesion in the secondary minimum of the potential energy diagram where separation will be observed between substratum and plasmalemma. If the same results could be extended to cell to cell adhesion this is also consistent with the relative weakness of the adhesions and also with the penetrability of tracer molecules into the adhesion. Cellsubstratum adhesions are also relatively weak. Secondary minimum type adhesions imply that at close separations repulsive force exceeds the force of attraction. If this were not so there would be no clear separations Separation distances of 5 to $10 \mathrm{~nm}$ are typical of secondary minimum adhesions. This type of adhesion is also consistent with a relatively long range force of attraction that exceeds that of repulsion at an appreciable distance from the surface. It has been usual to identify the repulsive force as being due to electrostatic forces of repulsion.

This type of result is not inconsistent with there being molecular bridges between the two surfaces but it is highly inconsistent with there being a high density of such bridging molecules. If there were the refractive index of the intervening gap between cell and substratum would be higher and the IRM image nature would change markedly towards the type of intensity that would be experienced where there was only a single reflection. This would not show evidence of variation with thickness because there would be no sec- ond deeper reflection at the cell/intercellular material interface to set up the interference. If there was a gently gradient of refractive index increase as the plasmalemma was approached the images obtained would be unsharp.

\section{Reactions of the Cell to Micro- and to Nanotopography}

It is interesting that IRM and TIRFM show no evidence of a special reaction of the cell to the substratum of surfaces bearing microgrooves. Adhesion experiments suggest that adhesion is stimulated by encounter with topographical boundaries (Curtis and Clark, 1990) but these do not show as changes in cell-substratum separation distance as seen by either of these methods of microscopy. Such observations can only of course be carried out on very shallow grooves. On deeper grooves the cell normally bridges from side to side and the cell is too far away from the surface to yield an IRM image. TIRFM images show that the cell is far away from the substratum in such regions. On grooves that are nanometrically deep the cells appear to conform to the surface but not to change their general pattern of adhesion at this point. The groove edges are not seen in these images. Reactions caused by contact with the edges such as changes in cell orientation or tyrosine kinase activation are visible by other forms of microscopy or by an increase in the area of closer contacts. These findings suggest that many topographies do not have special areas of high adhesion sufficient to attract the cell closer but that they signal to the cells to change their cytoskeletal behaviour. For example this might occur through the substrate allowing the cell to stretch more in one direction than another due to its shape.

There is, however, a difficulty in this interpretation because indicators of focal contacts such as vinculin localisation lie over the edges of the topography. The question then arises as to whether the optical conditions at a discontinuity are such that IRM images are destroyed.

\section{Fast Lifetime}

When these methods are applied to the development of adhesions they reveal interesting, previously apparently, unreported behaviour. A contact will suddenly appear as a very small area of image. This may appear and disappear, presumably as Brownian motion or other forces move this part of the cell towards or away from the surface. If the formation of the adhesion continues the area of contact visible by interference increases and becomes darker in places suggesting that the cell-substratum gap is thinning.

\section{Tapping}

In some cells, especially neurons at the growth cone, microspikes can be observed to tap the surface with a frequency I have measured from recordings of at least $10 \mathrm{~Hz}$. It is interesting that Fromherz (1997) predicted such behaviour on the basis of ion channel fluctuations altering the micro-environment close to the plasmalemma so that electrostatic forces of repulsion are changed, Drazba et al. (1997) and Gunderson (1988) described the static closeness of approach of microspikes on cadherin surfaces. 


\section{Time scale}

One important fact that can be derived from this type of observation is that an adhesion, sufficient to hold the cell in place can form in a few seconds or possibly still more rapidly, see Figure 6 . The attachments usually start at a single site and in a few instances, such as the attachment of primary chondrocytes remain very small in area.

\section{Drainage}

As the cell settles onto the substratum the medium between them drains away to the surroundings. Theoretical reasoning (Elton, 1948) suggests that the larger the area of fairly close approach (say closer than $1 \mu \mathrm{m}$ ) the longer and longer it will take the medium to drain away to give a contact distance of say $10 \mathrm{~nm}$. The time will rise linearly with the square of the area. Thus the system of having a number of point -like contacts such as the focal contact may ensure that the adhesion can form (and break) reasonably rapidly. If the cell is treated theoretically as a sphere sinking under gravity onto a surface the approach time to $10 \mathrm{~nm}$ separation is far longer than the observed time.

\section{References}

Axelrod D (1981) Cell-substrate contacts illuminated by total internal reflection fluoresence. J Cell Biol 89: 141145.

Braun D, Fromherz P (1997) Fluorescence interferencecontrast microscopy of cell adhesion on oxidized silicon. Appl Phys A 65: 341-348.

Braun D, Fromherz P (1998) Fluorescence interferometry of neuronal cell adhesion on micstructured silicon. Phys Rev Lett 81: 5241-5244.

Brightman MW, Klatzo I, Olsson Y, Reese TS (1970) The blood-brain barrier to proteins under normal and pathological conditions. J Neurol Sci 10: 215-239.

Burmeister JS, Olivier LA, Reichert WM, Truskey GA (1998) Application of total internal reflection fluorescence microscopy to study cell adhesion to biomaterials. Biomaterials 19: 307-325.

Curtis ASG (1964) The mechanism of adhesion of cells to glass. J Cell Biol 20: 199-215.

Curtis ASG (1989) Interference reflection microscopy. In: Light Microscopy in Biology: a Practical Approach. Lacey AJ (ed). IRL Press, Oxford. pp. 45-49.

Curtis, ASG, Clark P (1990) The effects of topographic and mechanical properties of materials on cell behaviour. Crit Rev Biocompatibility 5: 343-362.

Curtis, ASG, Wilkinson CDW (1998) Reactions of cells to topography. J Biomater Sci 9: 1313-1329.

DePasquale JA, Izzard CS (1987) Evidence for an actin-containing cytoplasmic precursor of the focal contact and the timing of incorporation of vinculin at the focal contact. J Cell Biol 105: 2803-2809.

Drazba J, Liljelund P, Smith C, Payne R, Lemmon V
(1997) Growth cone interactions with purified cell and substrate adhesion molecules visualized by interference reflection microscopy. Devel Brain Res 100: 183-197.

Elton GAH (1948) Electroviscosity. I The flow of liquids between surfaces in close proximity. Proc Roy Soc London A 194: 259-274.

Förster T (1949) Experimentele und theoretische Untersuchung des zwischenmolekularen Uebergangs von Elektronenanregungsenergie (Experimental and theoretical investigation of the intermolecular transition of electron excitation energy). Z Naturforsch 4a: 321-327.

Fromherz P (1997) Self-gating of ion channels in cell adhesion. Phys Rev Lett 78: 4131-4134.

Giebel KF, Bechinger C, Herminghaus S, Riedel M, Leiderer P, Weiland U, Bastmeyer M (1999) Imaging of cell/substrate contacts of living cells with surface plasmon resonance microscopy. Biophys J 76: 509-516.

Gingell D, Todd I (1979) Interference reflection microscopy: a quantitative theory for image interpretation and its application to cell-substratum separation measurement. Biophys J 26: 507-526.

Gingell D, Todd I, Bailey J (1985) Topography of cellglass apposition revealed by total internal reflection fluorescence of volume markers. J Cell Biol 100: 1334- 1338, 677-693.

Gundersen RW (1988) Interference reflection microscopic study of dorsal root growth cones on different substrates: Assessment of growth cone-substrate contacts. J Neurosci Res 21: 298-306.

Hunter RJ (1997) Foundations of Colloid Science. 674+xii pp. Clarendon Press, Oxford.

Izzard CS, Lochner LR (1976) Cell-to-substrate contacts in living fibroblasts: an interference reflexion study with an evaluation of the technique. J Cell Sci 21: 129159.

Richards RG, ap Gwynn I (1995) Backscattered electron imaging of the undersurface of resin-embedded cells by field emission scanning electron microscopy. J Microsc 177: 43-52.

Richards RG, ap Gwynn I, Bundy KJ, Rahn BA (1995) Microjet impingement followed by scanning electron microscopy as a qualitative technique to compare cellular adhesion to various biomaterials. Cell Biol Int 19: 10151024.

Usson Y, Guignandon A, Laroche N, Lafage-Proust MH, Vico L (1997) Quantitation of cell-matrix adhesion using confocal image analysis of focal contact associated proteins and interference reflection microscopy. Cytometry 28: 298-304.

Verschueren H (1985) Interference reflection microscopy in cell biology: Methodology and applications. J Cell Sci 75: 279-301.

Vickery SA, Dunn RC (1999) Scanning near-field fluorescence resonance energy transfer microscopy. Biophys J 76: 1812-1818. 